\title{
Color Registration of Underwater Images for Underwater Sensing with Consideration of Light Attenuation
}

\author{
Atsushi Yamashita, Megumi Fujii and Toru Kaneko
}

\begin{abstract}
Colors of objects observed in underwater environments are different from those in air. This is because the light intensity decreases with the distance from objects in water by light attenuation. Robots on the ground or in air usually recognize surrounding environments by using images acquired with cameras. The same is / will be true of underwater robots. However, recognition methods in air based on image processing techniques may become invalid in water because of light attenuation. Therefore, we propose a color registration method of underwater images. The proposed method estimates underwater environments where images are acquired, in other words, parameters essential to color registration, by using more than two images. After estimating parameters, color registration is executed with consideration of light attenuation. The effectiveness of the proposed method is verified through experiments.

key words: underwater sensing, color registration, light attenuation, computer vision, image processing
\end{abstract}

\section{INTRODUCTION}

In this paper, we propose a color registration method of underwater images with consideration of light attenuation.

In recent years, demands for underwater tasks, such as digging of ocean bottom resources, exploration of aquatic environments, rescues, and salvages, have increased. Therefore, underwater robots or underwater sensing systems that work instead of human become important, and technologies for observing underwater situations correctly and robustly from cameras of these systems are needed [1]. However, it is very difficult to observe underwater environments with cameras [2]-[4], because of the following three big problems.

1) View-disturbing noises (Fig. 1(a))

2) Light refraction effects (Fig. 1(b))

3) Light attenuation effects (Fig. 2(a)(b))

The first problem is about suspended matters, such as bubble noises, small fishes, and small creatures. They may disturb camera's field of view (Fig. 1(a)).

The second problem is about the refraction effects of light. If cameras and objects are in the different condition where the refraction index differs from each other, problems do occur and a precise measurement cannot be achieved. For example, Fig. 1(b) shows an image of a single rectangular object when water is filled to the middle. In this case, the size and the shape of the object look different between above and below the water surface. Therefore, it becomes difficult to

A. Yamashita, M. Fujii and T. Kaneko are with Department of Mechanical Engineering, Shizuoka University, 3-5-1 Johoku, Hamamatsu-shi, Shizuoka 432-8561, JAPAN (e-mail: yamashita@ieee.org)

A. Yamashita is now with Department of Mechanical Engineering, California Institute of Technology, 1200 E. California Blvd. MC 104-44, Pasadena, CA 91125, USA (e-mail: atsushi@caltech.edu)

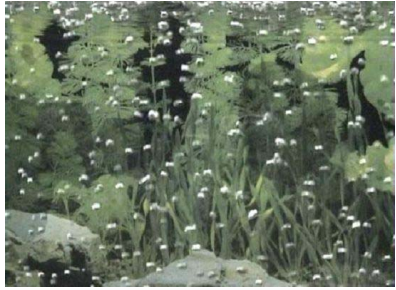

(a) View-disturbing noises.

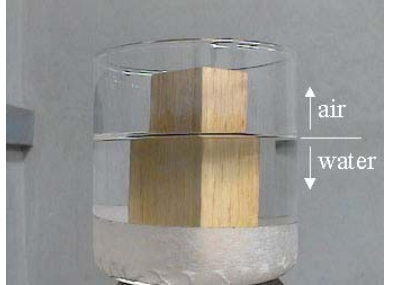

(b) Refraction effects
Fig. 1. Examples of aquatic images 1.

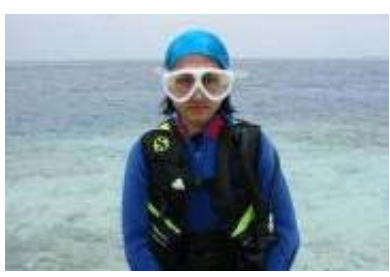

(a) Image in air.



(b) Image in water.
Fig. 2. Examples of aquatic images 2.

measure precise positions and shapes of objects when water exists because of the image distortion by the refraction of the light.

The third problem is about the attenuation effects of light. The light intensity decreases with the distance from objects in water by light attenuation depending on the wavelength of light. Red light decreases easier than blue light in water [2]. For example, there exists red color at the neck of the person in Fig. 2(a). However, in the aquatic environment, red color is attenuated and cannot be observed in Fig. 2(b), although blue color can be observed. In this way, colors of objects observed in underwater environments are different from those in air. Therefore, it becomes difficult to classify or to recognize objects in water by observing their colors and textures.

As to the first and second problems, there are several methods that can work well in aquatic environments [5] [11]. These previous studies remove adherent noises from images by image processing techniques or consider the light refraction effects when measuring three dimensional (3-D) shapes and positions of objects in water.

However, as to the third problem, there are few methods that can investigate objects' colors in aquatic environments where different colors are observed by light attenuation effects from aerial environments. For example, a navigation method of autonomous underwater vehicles based on arti- 
ficial underwater landmarks is proposed [12], however, this technique uses only landmarks whose colors are less subject to light attenuation effects in order to avoid light attenuation problems. An underwater image mosaicing method [13] or an image processing technique for recognizing acanthaster by color [14] are also proposed. However, these techniques assume that aquatic environments are same with aerial environments, although there is a risk of misjudgment in consequence of light attenuation effects.

In fact, a lot of researches in the fields of computer vision or computer graphics are studying about objects' appearance in air (e.g. [15]-[18]), because "colors" or "textures" have important and necessary information about objects. Of course, the same is true of aquatic environments. For example, undersea remains or abyssal creatures must be investigated in water, because they cannot be pulled up out of the sea and their images must be taken in water.

Therefore, in this paper, we propose a color registration method of underwater images. The proposed method estimates underwater environments where images are acquired, in other words, parameters essential to color registration, by using more than two images. After estimating parameters, color registration is executed with consideration of light attenuation.

The composition of this paper is detailed below. In Section II, the principle of the light attenuation and observed colors in liquid are explained. In Section III, we propose color registration method. Section IV mentions about experiments and Section V describes conclusions.

\section{COLOR IN LIQUid}

\section{A. Light Attenuation in Liquid}

As described above, colors of objects observed in underwater environments are different from those in air. This is because the light intensity decreases with the distance from objects in liquid by light attenuation depending on the wavelength of light. Figure 3 shows the light attenuation in water. For example, the intensity of red color decreases to about a half at the point whose distance from the light source is $2 \mathrm{~m}$, although that of blue color hardly changes. Red color disappears in $20 \mathrm{~m}$ distance. In this way, colors of objects in water looks different from those in air.

Equation (1) can describe these phenomena (Fig. 4).

$$
L_{i}(z)=L_{0, i} \exp \left(-c_{i} z\right)
$$

where $i$ is the wavelength of light, $z$ is the distance between the light source and the viewpoint, $L_{i}(z)$ is the light intensity of wavelength $i, L_{0, i}$ is the light intensity of wavelength $i$ at the light source, and $c_{i}$ is attenuation coefficient (or extinction coefficient) of liquid at wavelength $i$, respectively.

Attenuation coefficient consists of absorption coefficient and scattering coefficient, because light attenuation consists of light absorption and light scattering. Figure 5 shows attenuation coefficient of water. Attenuation coefficient of water changes very much with the wavelength of light. Consequently, observed colors changes in underwater environments.

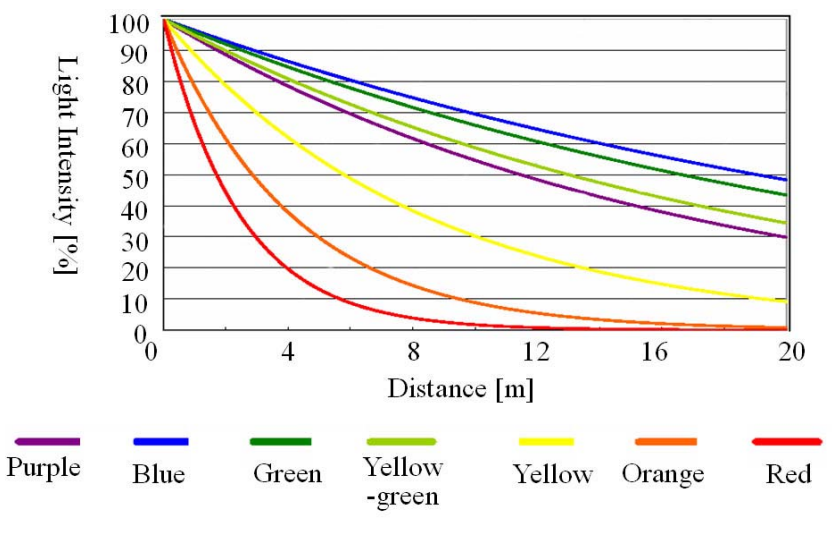

Fig. 3. Light intensity in water.

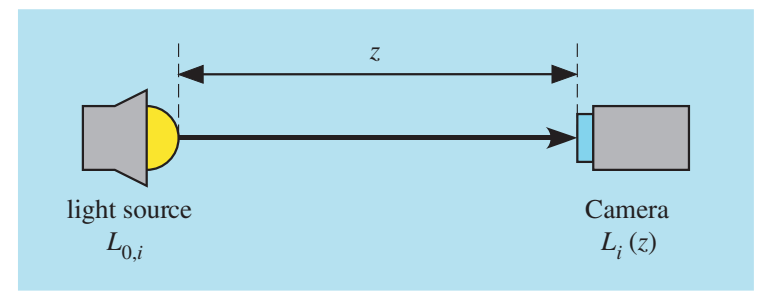

Fig. 4. Light attenuation in liquid.

\section{B. Light Reflection in Liquid}

The light travels from the light source, is reflected at the object surface, and finally arrives at the viewpoint (Fig. 6). Therefore, observed colors of objects at the viewpoint are under the influence of the light intensity of light source, properties of object surfaces, and traveling distance of the light.

The diffuse reflection of light in air where the light attenuation does not exist is expressed as follows;

$$
I_{i}(l, z)=\frac{L_{0, i} \kappa_{i} \cos ^{3} \alpha}{z^{2}},
$$

where $l$ is the distance between the object surface and the viewpoint, $z$ is the distance between the light source and the object surface, $I_{i}(l, z)$ is the light intensity of wavelength $i$,

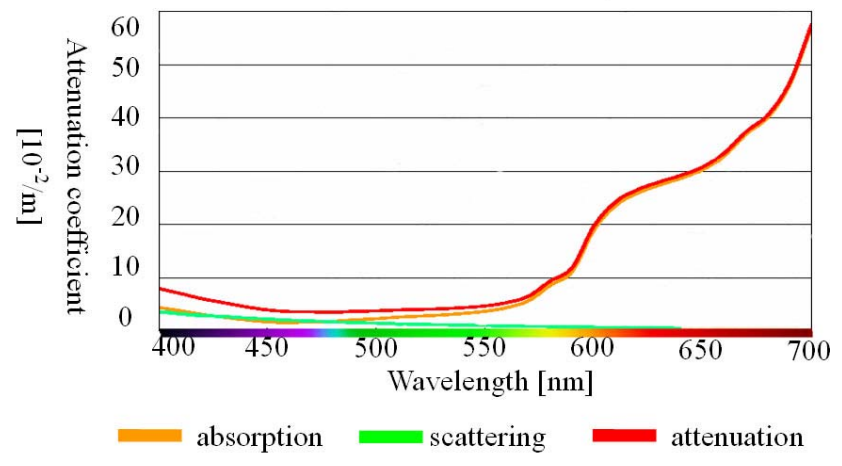

Fig. 5. Attenuation coefficient of water. 


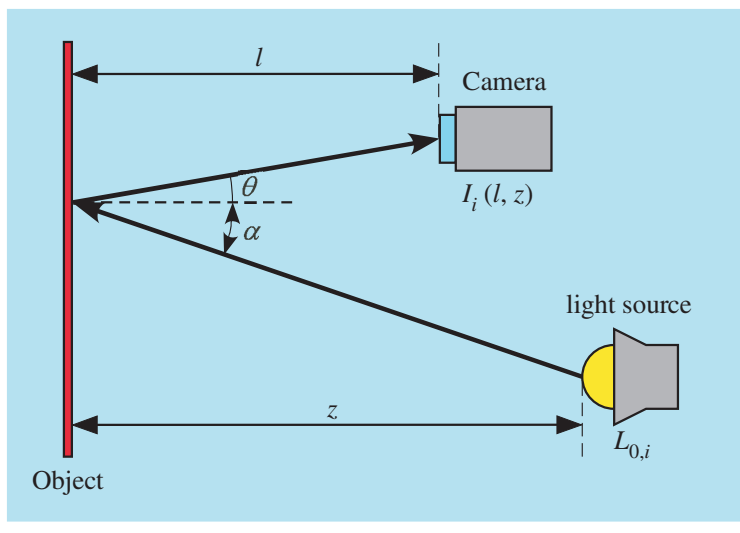

Fig. 6. Light refraction in liquid.

$\kappa_{i}$ is reflectance ratio of the object's surface of wavelength $i$, and $\alpha$ is the angle between the ray vector of light from the light source and the normal vector of object's surface, respectively.

The light intensity $I_{i}(l, z)$ describes observed colors of objects in air.

The difference between observed colors of objects in liquid and those in air is whether there are light attenuation effects or not. Therefore, the light intensity $I_{i}(l, z)$ in liquid can be expressed as follows by considering the diffuse reflection of light in liquid from Eq.(1) and (2) (Fig. 6).

$$
\begin{aligned}
I_{i}(l, z)= & \frac{L_{0, i} \kappa_{i} \cos ^{3} \alpha}{z^{2}} \\
& \exp \left\{-c_{i}\left(\frac{z}{\cos \alpha}+\frac{l}{\cos \theta}\right)\right\},
\end{aligned}
$$

where $\theta$ is the angle between the ray vector of light from the object and the optical axis of lens.

We can calculate the light intensity in arbitrary conditions by using Eq.(3) when all parameters are known. In other words, we can generate images in arbitrary conditions in that case.

However, some of these parameters are unknown in almost all cases. We usually have to use special measuring devices in a well-equipped laboratory and acquire a large mount of data when measuring all parameters with high accuracy (e.g. [19]). In particular, reflectance ratio of objects and light intensity of the light source are difficult to measure. It is distant in aquatic environments in practice.

On the other hands, color registration of acquired image(s) is realistic and effective way.

\section{Color Registration Method}

\section{A. Relationship between Two Images}

The relationship between light intensity $I_{i}\left(l, z, c_{i}\right) \quad(i$ : wavelength, $l$ : distance between the object surface and the viewpoint, $z$ : distance between the light source and the object surface, $c_{i}$ : attenuation coefficient of liquid) and light intensity $I_{i}\left(l^{\prime}, z^{\prime}, c_{i}^{\prime}\right)$ at another position and in another liquid (or air) can be expressed as follows;

$$
\begin{aligned}
I_{i}\left(l^{\prime}, z^{\prime}, c_{i}^{\prime}\right)= & I_{i}\left(l, z, c_{i}\right)\left(\frac{z}{z^{\prime}}\right)^{2} \frac{\cos ^{3} \alpha^{\prime}}{\cos ^{3} \alpha} \\
& \exp \left\{-c_{i}^{\prime}\left(\frac{z^{\prime}}{\cos \alpha^{\prime}}+\frac{l^{\prime}}{\cos \theta^{\prime}}\right)\right. \\
& \left.+c_{i}\left(\frac{z}{\cos \alpha}+\frac{l}{\cos \theta}\right)\right\} .
\end{aligned}
$$

Reflectance ratio of the object's surface (object properties) and the light intensity of the light source (light source properties) do not appear in Eq.(4). Therefore, colors of objects in air (images acquired in air) can be calculated from images acquired in liquid when the geometrical relationship between two conditions is known.

\section{B. Estimation of Unknown Parameters}

In addition, colors of objects in air can be estimated from images acquired in liquid even when the condition of image acquisition is unknown, by using more than two images acquired in liquid at more than two places; position $\mathrm{A}$ and position B. This is because the conditions (unknown parameters) of image acquisitions in liquid can be also estimated if the relative geometrical relationship (distance) between position $\mathrm{A}$ and position $\mathrm{B}$ is known.

Attenuation coefficients $c_{i, A}$ equal to $c_{i, B}\left(c_{i, A}=c_{i, B}=\right.$ $c_{i}$ ) when only the places of image acquisitions differ from each other. In this case, the relationship between two images is expressed as follows;

$$
\begin{aligned}
\frac{I_{i, B}(u, v)}{I_{i, A}(u, v)}=\left(\frac{z_{A}}{z_{B}}\right)^{2} \frac{\cos ^{3} \alpha_{B}}{\cos ^{3} \alpha_{A}} \exp & \left\{c _ { i } \left(\frac{z_{A}}{\cos \alpha_{A}}+\frac{l_{A}}{\cos \theta_{A}}\right.\right. \\
& \left.\left.-\frac{z_{B}}{\cos \alpha_{B}}-\frac{l_{B}}{\cos \theta_{B}}\right)\right\},
\end{aligned}
$$

where $(u, v)$ is image coordinate, and $I_{i, A}(u, v)$ and $I_{i, B}(u, v)$ are acquired images at position $\mathrm{A}$ and position $\mathrm{B}$, respectively.

We usually take underwater images by a camera with photoflash. In this case, the viewpoint corresponds to the camera (optical center or lens center in a precise sense), and the light source corresponds to flash of the camera. Therefore, the relative relationship between the light source and the viewpoint never changes when the camera position changes. The relative geometrical relationship between the light source and the viewpoint can be easily estimated by conventional camera calibration techniques (e.g. [20]).

Here, let vector $\mathbf{d}$ be the geometrical relationship between position A and position B. The left side of Eq.(5) can be calculated from two acquired image. All the parameters of the right side of Eq.(5) can be expressed by using $\mathbf{d}$ and only one parameter $\left(e . g . l_{A}\right)$ when geometrical relationship between the light source and the viewpoint is known.

Consequently, the number of unknown parameters equals to one $\left(e . g . l_{A}\right)$, if the movement of camera $\mathbf{d}$ and attenuation coefficient $c_{i}$ are known, and this one unknown parameter can be gained by solving Eq.(5). After that, all parameters of the right side of Eq.(5) can be also calculated by using $\mathbf{d}$ and the obtained one unknown parameter. 
Accordingly, we can estimate all unknown parameters of right side of Eq.(4) that are essential to color registration $(l, z, \alpha, \theta)$. We can recover new images taken at another places in same liquid from acquired one image (image $\mathrm{A}$ or image B) by substituting arbitrary values to $l^{\prime}, z^{\prime}, \alpha^{\prime}, \theta^{\prime}$ in Eq.(4). New images acquired in air also can be estimated by substituting $c_{i}=0$ in Eq.(4) in place of attenuation coefficient of liquid.

Above mentioned analysis is approved under the assumption that environment light does not exist. In aquatic environment, the influence of environment light is very little because sunlight is also attenuated. If there is strong environment light, the influence of environment light can be easily canceled by acquiring two images with and without flash of a camera at the same place with a subtraction method (e.g. [21]).

\section{EXPERIMENT}

\section{A. Experimental Condition}

We acquired underwater images in liquid by using the digital camera NIKON D70 that can save images with RAW format. The light intensity values can be extracted by using RAW images, while we cannot do so by using JPEG or BMP images. This camera has three color filters; R (red), G (green), and B (blue). We assume that only the light whose wavelength is $700 \mathrm{~nm}$ (red) ${ }^{1}, 546 \mathrm{~nm}$ (green), and $436 \mathrm{~nm}$ (blue) can pass these filters, respectively, and the light of different wavelength cannot pass them. The range of the light intensity that the camera can measure is from 0 to 4095 .

Water whose attenuation coefficients of red, green, and blue colors are $0.4000,0.0391$, and 0.0348 , respectively, was adopted as liquid [2]. Attenuation coefficients of every wavelength in air was set zero.

Several planar paintings were placed in the water tank filled in water, and images of these paintings were acquired with the camera.

\section{B. Color Registration under Known Condition}

To verify the effectiveness of color registration method, underwater images were taken under the condition that geometrical relationship between the camera and the object was known.

Figure 7 shows a color registration result of an underwater image (painting of a flower) with consideration of light attenuation when distance between the camera and the object changes.

Figure 7(a) shows the acquired image in water when the distance between the camera and the object is $0.80 \mathrm{~m}$, and Fig. 7(b) shows the color registration result under the condition that the distance between the camera and the object is $0.70 \mathrm{~m}$. Figure $7(\mathrm{~b})$ is generated from Fig. 7(a) by using Eq.(4). For comparison, we also acquired the underwater image when the distance between the camera and the object

\footnotetext{
${ }^{1}$ It is anticipated that the use of the absorption coefficient at $700 \mathrm{~nm}$ would overestimate the recovery of the $\mathrm{R}$ channel, because the transmittance of commercial digital cameras at $700 \mathrm{~nm}$ is usually low due to an infrared rejection filter.
}

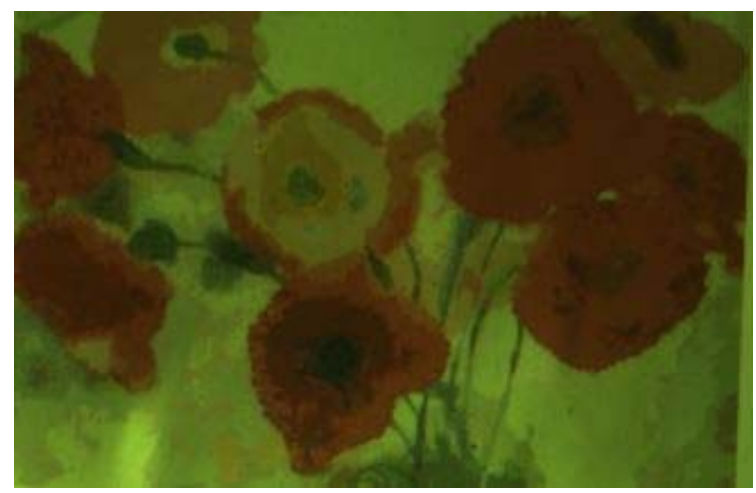

(a) Observed image in water.

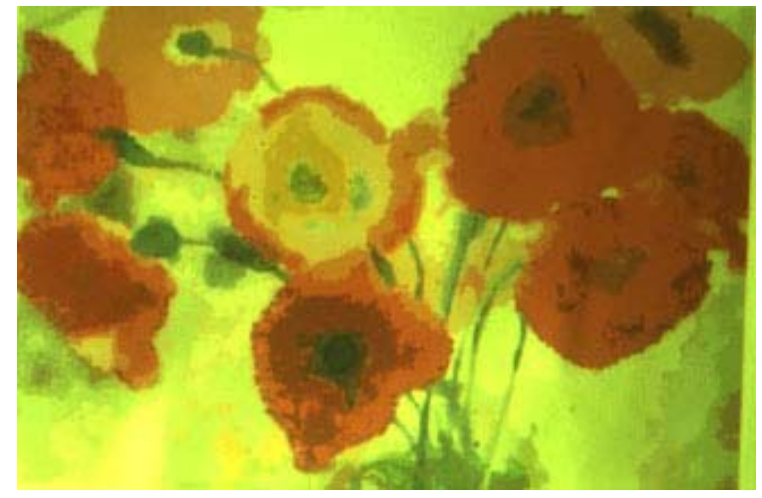

(b) Color registration result.

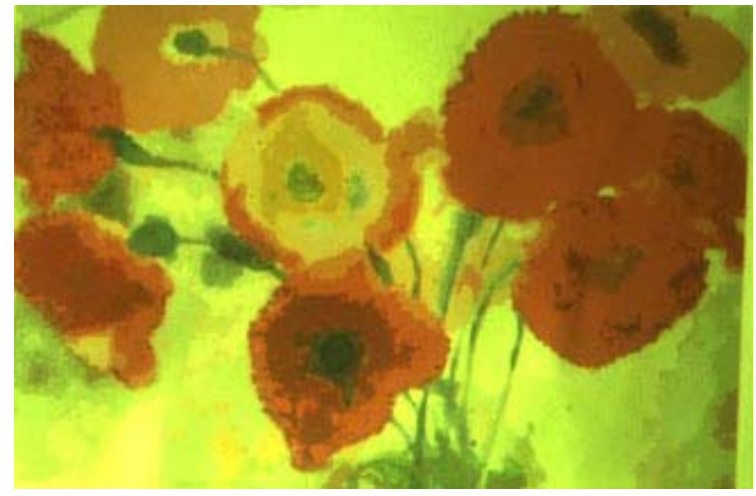

(c) Observed image at different location.

Fig. 7. Color registration results 1 .

is $0.70 \mathrm{~m}$ (Fig. $7(\mathrm{c})$ ). The appearance of Fig. $7(\mathrm{~b})$ is very similar to that of Fig. 7(c), although those of Fig. 7(a) and Fig. 7(b) differ from each other.

Figure 8 shows another result (painting of a "kimono"2 lady) when both distance between the camera and the object and surrounding medium change.

Figure 8(a) shows the acquired image in water when the distance between the camera and the object is $0.80 \mathrm{~m}$. Figure 8(b) shows the color registration image in air when the distance between the camera and the object is $0.60 \mathrm{~m}$, that is also generated by using Eq.(4) from Fig. 8(a). For

\footnotetext{
${ }^{2}$ Kimono is a long, loose traditional Japanese robe with wide sleeves, tied with a sash.
} 




(a) Observed image in water.

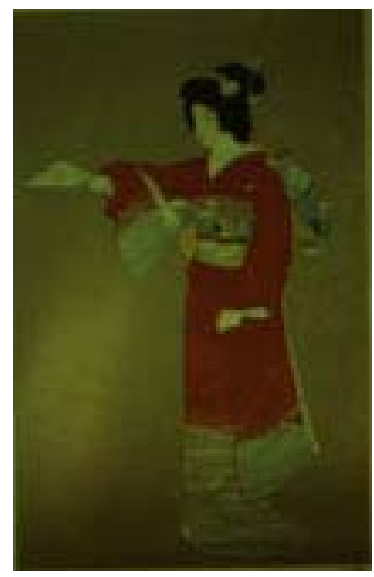

(b) Color registration result.

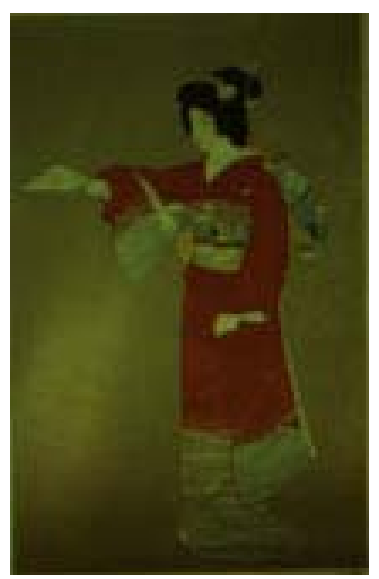

(c) Observed image in air.

Fig. 8. Color registration results 2 .

comparison, we also acquired the image in air when the distance between the camera and the object is $0.60 \mathrm{~m}$ (Fig. 7(c)).

Colors of the corrected image (Fig. 8(b)) is clear, while the original acquired image in water (Fig. 8(a)) has grave colors because of light attenuation. It is verified that the color registration can be executed fairly when comparing the corrected image with the image in air under the same lighting condition (Fig. 8(c)).

\section{Color Registration under Unknown Condition}

Figure 9 shows the color registration result under the unknown condition.

We also acquired two images (painting of a cat) in water (Fig. 9(a)(b)), estimated the image acquisition condition, and reconstructed the image in air under the same lighting condition (Fig. 9(c)) from images in water.

The distance between the camera and the object was 0.80m when Fig. 9(a) was taken. The distance between the camera and the object was estimated by using two images at the different positions (Fig. 9(a) and (b)). Estimated distance is $0.82 \mathrm{~m}$ when using red color data, $0.78 \mathrm{~m}$ when using green color data, and $0.78 \mathrm{~m}$ when using blue color
TABLE I

LIGHT INTENSITY RGB RATIO.

\begin{tabular}{l|c|c|c}
\hline & $\mathrm{R}$ & $\mathrm{G}$ & $\mathrm{B}$ \\
\hline (a) Observed colors in water 1 (Fig. 9(a)). & 0.95 & 1.00 & 0.29 \\
\hline (c) Color registration results (Fig. 9(c)). & 1.41 & 1.00 & 0.29 \\
\hline (d) Observed colors in air (Fig. 9(d)). & 1.45 & 1.00 & 0.29 \\
\hline
\end{tabular}

data, respectively. In consequence, the distance between the camera and the object is calculated as $0.793 \mathrm{~m}$ by averaging, while the true value is $0.8 \mathrm{~m}$. From this result, it is verified that our method can estimate the conditions when underwater images are acquired by using more than two images.

After estimating the conditions, color registration of the image is done (Fig. 9(c)) by considering estimated conditions. Colors of the corrected image (Fig. 9(c)) are similar to those of image taken in air (Fig. 9(d)). Table I shows quantitative results about the color ratio of red $(\mathrm{R})$ : green (G: we set 1.00) : blue (B). The ratio of the corrected image is very similar to that of the image in air, while the ratio of original image is differ from that of the image in air.

From these results, the validity of the proposed method was verified.

\section{CONCLUSION}

We propose a color registration method of underwater images with consideration of light attenuation. The proposed method estimates parameters essential to color registration, by using more than two images. After estimating parameters, colors of these images can be corrected with consideration of light attenuation.

The effectiveness of the proposed method is verified through experiments.

As the future works, classification or recognition method of objects in underwater images that are under the influence of light attenuation, and cannot classify or recognize them without color registration. A stereo camera system is also adopted for the robust color registration and 3-D measurement of objects in underwater environments.

\section{ACKNOWLEDGEMENT}

The authors would like to thank Prof. Kazunori Umeda and Mr. Naoya Ogawa of Chuo University for helping RAW image analysis of NIKON D70. The authors also would like to thank Prof. Joel W. Burdick of California Institute of Technology and his laboratory members for useful suggestions.

This research was partially supported by the Ministry of Education, Culture, Sports, Science and Technology, Grantin-Aid for Scientific Research (C), 14550416, 2002.

\section{REFERENCES}

[1] Junku Yuh and Michael West: "Underwater Robotics," Advanced Robotics, Vol.15, No.5, pp.609-639, 2001.

[2] E. O. Hulburt: "Optics of Distilled and Natural Water," Journal of the Optical Society of America, Vol.35, pp.689-705, 1945.

[3] W. Kenneth Stewart: "Remote-Sensing Issues for Intelligent Underwater Systems," Proceedings of the 1991 IEEE Computer Society Conference on Computer Vision and Pattern Recognition (CVPR1991), pp.230-235, 1991. 
[4] Frank M. Caimi: Selected Papers on Underwater Optics, SIPE Milestone Series, Vol.MS118, 1996.

[5] Hideo Saito, Hirofumi Kawamura and Msasato Nakajima: "3D Shape Measurement of Underwater Objects Using Motion Stereo," Proceedings of 21th International Conference on Industrial Electronics, Control, and Instrumentation, pp.1231-1235, 1995.

[6] Rongxing Li, Haihao Li, Weihong Zou, Robert G. Smith and Terry A. Curran: "Quantitive Photogrammetric Analysis of Digital Underwater Video Imagery," IEEE Journal of Oceanic Engineering, Vol.22, No.2, pp.364-375, 1997.

[7] Atsushi Yamashita, Etsukazu Hayashimoto, Toru Kaneko and Yoshimasa Kawata: "3-D Measurement of Objects in a Cylindrical Glass Water Tank with a Laser Range Finder," Proceedings of the 2003 IEEE/RSJ International Conference on Intelligent Robots and Systems (IROS2003), pp.1578-1583, 2003.

[8] Atsushi Yamashita, Hirokazu Higuchi, Toru Kaneko and Yoshimasa Kawata: "Three Dimensional Measurement of Object's Surface in Water Using the Light Stripe Projection Method," Proceedings of the 2004 IEEE International Conference on Robotics and Automation (ICRA2004), pp.2736-2741, 2004.

[9] Hayato Kondo, Toshihiro Maki, Tamaki Ura, Yoshiaki Nose, Takashi Sakamaki and Masaaki Inaishi: "Relative Navigation of an $\mathrm{Au}-$ tonomous Underwater Vehicle Using a Light-Section Profiling System," Proceedings of the 2004 IEEE/RSJ International Conference on Intelligent Robots and Systems (IROS2004), pp.1103-1108, 2004.

[10] Atsushi Yamashita, Shinsuke Ikeda and Toru Kaneko: "3-D Measurement of Objects in Unknown Aquatic Environments with a Laser Range Finder," Proceedings of the 2005 IEEE International Conference on Robotics and Automation (ICRA2005), pp.3923-3928, 2005.

[11] Atsushi Yamashita, Susumu Kato and Toru Kaneko: "Robust Sensing against Bubble Noises in Aquatic Environments with a Stereo Vision System," Proceedings of the 2006 IEEE International Conference on Robotics and Automation (ICRA2006), pp.928-933, 2006.

[12] Son-Cheol Yu, Tamaki Ura, Teruo Fujii and Hayato Kondo: "Navigation of Autonomous Underwater Vehicles based on Artificial Underwater Landmarks," Proceedings of Oceans'01, Vol.1, pp.409-416, 2001.

[13] Hiroshi Sakai, Toshinari Tanaka, Satomi Ohata, Kazuo Ishii and Tamaki Ura: "Underwater Image Mosaicing Using Underwater Robot," Proceedings of the 2004 JSME Conference on Robotics and Mechatronics (ROBOMEC2004), 2A1-L2-27, pp.1-4, 2004, (in Japanese).

[14] Norimitsu Sakagami, Ssatoshi Tomita, Fumiaki Takemura and Shinichi Sagara: "A Study of Image Processing Technique for Recognizing Acanthaster," Proceedings of the 2006 JSME Conference on Robotics and Mechatronics (ROBOMEC2006), 1P1-E35, pp.1-3, 2006, (in Japanese).

[15] Yoichi Sato, Mark D. Wheeler, and Katsushi Ikeuchi: "Object Shape and Reflectance Modeling from Observation," ACM Transactions on Computer Graphics (Proceedings of SIGGRAPH 1997), pp.379-387, 1997.

[16] Stephen Robert Marschner: "Inverse Rendering for Computer Graphics," Doctoral Dissertation, Cornell University, 1998.

[17] Shoji Tominaga and Norihiro Tanaka: "Measuring and Rendering Art Paintings Using an RGB Camera," Annual Conference of the European Association for Computer Graphics (EUROGRAPHICS 2002), pp.299-306, 2002.

[18] Jinwei Gu, Chien-I Tu, Ravi Ramamoorthi, Peter Belhumeur, Wojciech Matusik and Shree Nayar: "Time-varying Surface Appearance: Acquisition, Modeling, and Rendering," ACM Transactions on Computer Graphics (Proceedings of SIGGRAPH 2006), pp.762-771, 2006.

[19] Paul Debevec, Andreas Wenger, Chris Tchou, Andrew Gardner, Jamie Waese and Tim Hawkins: "A Lighting Reproduction Approach to Live-Action Compositing," ACM Transactions on Computer Graphics (Proceedings of SIGGRAPH 2002), pp.547-556, 2002.

[20] Roger Y. Tsai: "A Versatile Camera Calibration Technique for HighAccuracy 3D Machine Vision Metrology Using Off-the-Shelf TV Cameras and Lenses," IEEE Journal of Robotics and Automation, Vol.RA-3, No.4, pp.323-344, 1987.

[21] Naoya Ogawa and Kazunori Umeda: "Range Measurement by a Digital Camera Using Flash," Proceedings of SPIE Vol.6051 ISOT Optomechatronic Machine Vision, pp.605108-1-605108-9, 2005.

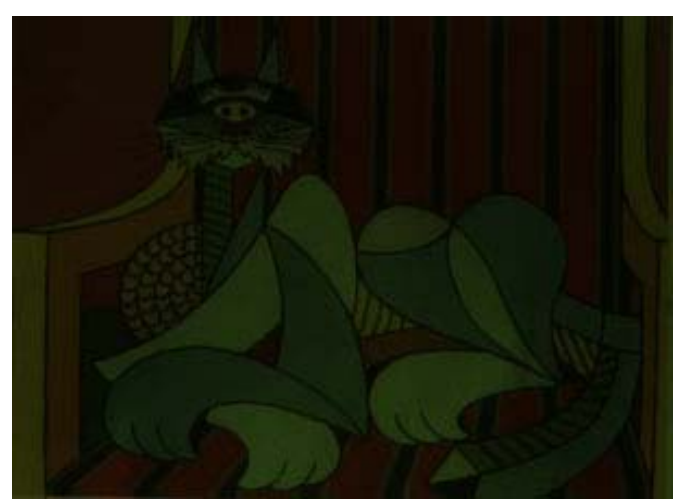

(a) Observed image in water 1 .

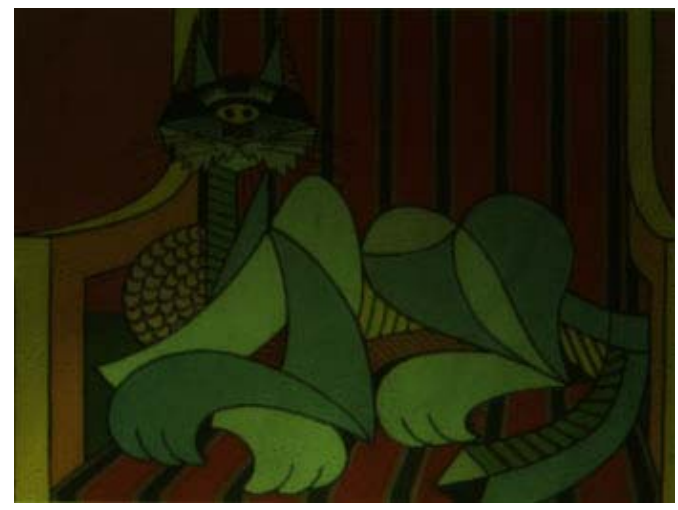

(b) Observed image in water 2 .

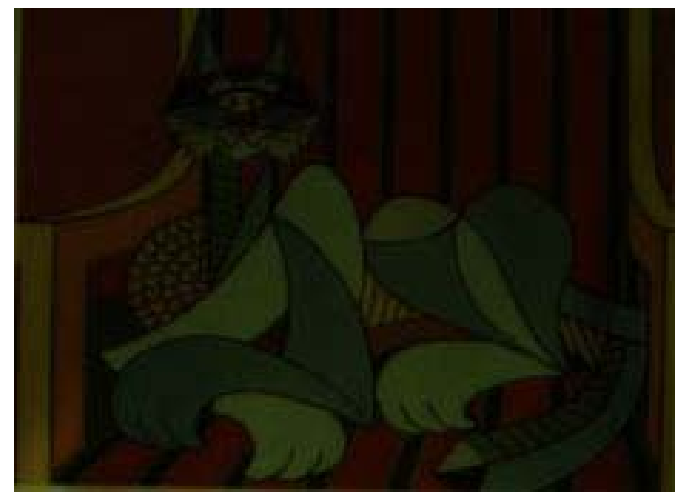

(c) Color registration result.

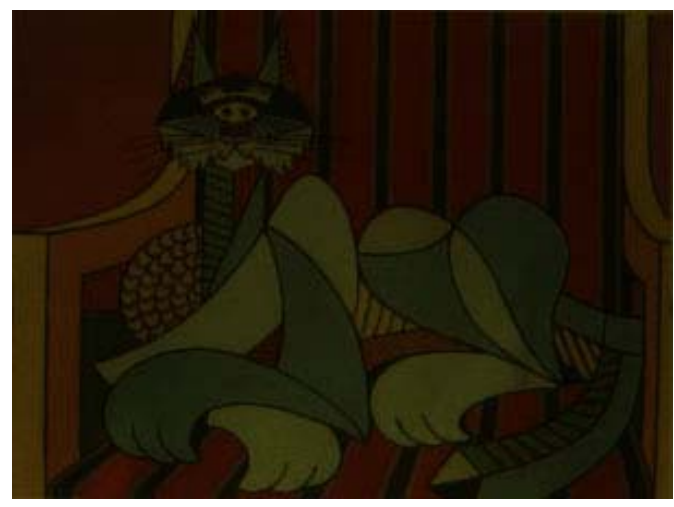

(d) Observed image in air.

Fig. 9. Color registration results 3 . 